\title{
Identification of 3 novel VHL germ-line mutations in Danish VHL patients
}

\author{
Mette Dandanell ${ }^{1}$, Lennart Friis-Hansen ${ }^{1}$, Lone Sunde ${ }^{2,3}$, Finn C Nielsen ${ }^{1}$ and Thomas v O Hansen ${ }^{1 *}$
}

\begin{abstract}
Background: von Hippel-Lindau (VHL) disease is a hereditary cancer syndrome in which the patients develop retinal and central nervous system hemangioblastomas, pheochromocytomas and clear-cell renal tumors. The autosomal dominant disease is caused by mutations in the VHL gene.

Methods: VHL mutational analysis was carried out by sequencing of the coding sequence and by multiplex ligation-dependent probe amplification analysis. The functional consequence of the variants was investigated using in silico prediction tools.

Results: A total of 289 probands suspected of having VHL syndrome have been screened for mutations in the VHL gene. Twenty-six different VHL mutations were identified in 36 families including one in-frame duplication, two frame-shift mutations, four nonsense mutations, twelve missense mutations, three intronic mutations and four large genomic rearrangements. Three of these mutations (c.319 C > T, c.342_343dupGGT and c.520_521dupAA) were novel.

Conclusions: In this study we report the VHL germ-line mutations found in Danish families. We found three novel VHL mutations where two were classified as pathogenic and the latter was classified as a variant of unknown significance. Together, our findings contribute to the interpretation of the potential pathogenicity of VHL germ-line mutations.
\end{abstract}

Keywords: von Hippel-Lindau disease, VHL, Germ-line mutations, Danish population

\section{Background}

von Hippel-Lindau (VHL) disease (OMIM 193300) is a dominantly inherited cancer syndrome characterized by central nervous system and retinal hemangioblastomas, clear cell renal carcinoma, neuroendocrine tumors and cysts of the pancreas, pheochromocytomas, endolymphatic sac tumors and/or papillary cystadenomas of the epididymis and broad ligament [1]. Patients without pheochromocytomas are classified as VHL type 1 and those with pheochromocytoma as VHL type 2. Type 2 patients are further divided into three subgroups: type $2 \mathrm{~A}$ patients with low risk of renal cell carcinomas, type $2 \mathrm{~B}$ patients with high risk of renal cell carcinomas and type $2 \mathrm{C}$ patients with isolated pheochromocytoma [2].

von Hippel-Lindau disease is caused by germ-line mutations in the $V H L$ tumor suppressor gene located on

\footnotetext{
* Correspondence: tvoh@rh.dk

${ }^{1}$ Center for Genomic Medicine, Rigshospitalet, Copenhagen University

Hospital, Copenhagen, Denmark

Full list of author information is available at the end of the article
}

the short arm of chromosome 3 (3p25-26). The gene spans $10 \mathrm{~kb}$, is composed of three exons (Figure 1) and encodes two proteins (pVHL19 and pVHL30) [3-5]. The complete VHL protein consists of 214 amino acids and has two structural domains: the $\alpha$-domain and the $\beta$-domain [6].

The best characterized function of pVHLs is its role as a substrate recognition component of the E3 ubiquitin protein ligase complex comprising pVHL, Elongin C, Elongin B, Cullin 2 and Rbx1. In this complex, pVHL targets the $\alpha$-subunit of hypoxia inducible factor 1 (HIF-1 $\alpha$ ) and hypoxia inducible factor 2 (HIF-2 $\alpha$, also known as EPAS1) transcription factors for ubiquitin-mediated proteolysis. The HIF transcription factors are involved in coordination of the cellular response to hypoxia and in transcriptional regulation of hypoxia inducible genes, including VEGF, $P D G F, T G F \alpha$ and EPO. pVHL inactivation leads to stabilization of HIF-1 and HIF-2 and subsequent transcriptional activation of HIF-induced target genes [2].

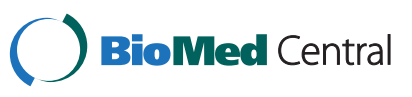




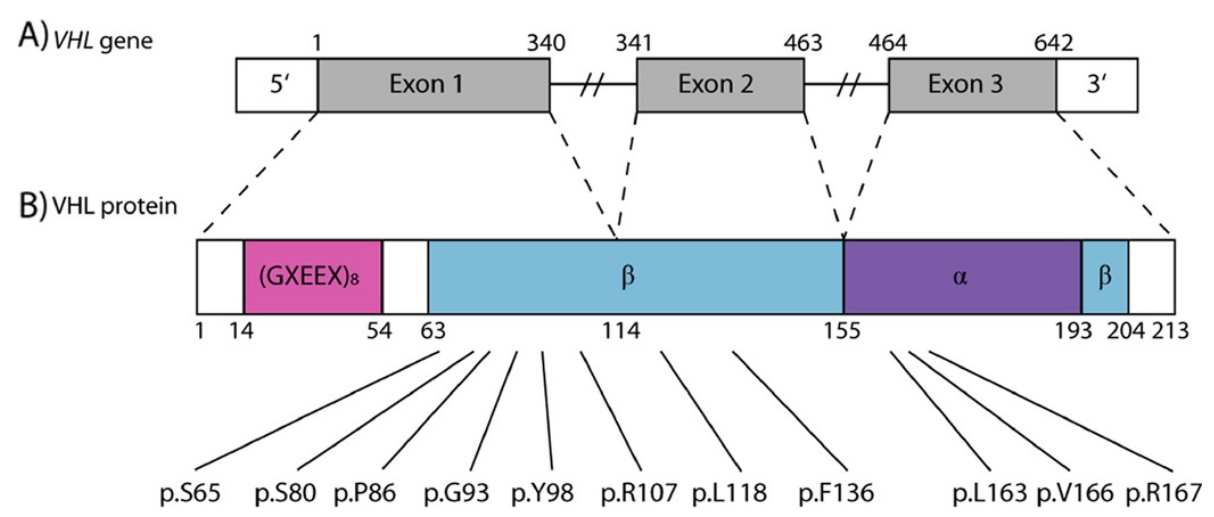

Figure 1 von Hippel-Lindau gene and protein structure. A) The VHL gene comprises three exons (gray). Nucleotide number is indicated above the gene structure. B) The VHL protein structure with (GXEEX) ${ }_{8}$ repeat motif (pink), a-domain (purple) and $\beta$-domain (blue). Codon number is indicated below the protein structure. The eleven residues subjected for missense mutations are indicated below the figure.

Since 1998 we have performed the screening of Danish patients suspected of having VHL disease. The clinical characterization of the Danish VHL mutation carriers has recently been published $[7,8]$ and in this study we report the $V H L$ germ-line mutations identified during this period.

\section{Methods}

\section{Patients}

The national Danish guidelines recommend mutational $V H L$ screening when a patient 1) exhibits at least two of the following VHL manifestations: familial history of renal cancers, paragangliomas, hemangioblastomas, endolymphatic sac tumors, neuroendocrine tumors, cysts of the pancreas and/or pheochromocytomas 2) has one VHL affected first degree relative and exhibits at least one of the mentioned manifestations [9]. Following verbal and written consent blood samples were collected from the probands. All family relatives are offered presymptomatic testing and genetic counseling.

\section{VHL screening}

Genomic DNA was purified from whole blood using either the QIAamp DNA mini kit (Qiagen, Hilden, Germany) or Maxwell 16 blood DNA purification kit (Promega, Stockholm, Sweden) according to manufacturer's instructions. The VHL exons were amplified using intronic primer pairs flanking each exon (Table 1) and the PCR products were subsequently sequenced using

Table 1 Forward and reverse primers used for PCR amplification of the $V H L$ gene

\begin{tabular}{lll}
\hline & Forward Primer & Reverse Primer \\
\hline Exon 1 & $5^{\prime}$-gcgcgcgaagactacggaggt-3' & 5'-gaatgctctgacgcttac-3' $^{\prime}$ \\
Exon 2 & $5^{\prime}$-gtggctctttaacaacctttgct-3' & 5'-cctgtacttaccacaacaaccttatc-3' $^{\prime}$ \\
Exon 3 & 5'-gtggacctagtctgtcactgagg-3' & 5'-agactcatcagtaccatcaaaag-3' \\
\hline
\end{tabular}

an ABI3730 DNA analyzer (Applied Biosystems, Foster City, CA). Moreover, genomic DNA was examined for large genomic rearrangements by multiplex ligationdependant probe amplification (MLPA) analysis using the salsa MLPA P016 VHL kit (MRC-Holland, Amsterdam, Holland). All sequence variants were verified in an independent blood sample. VHL variants are numbered according to accession number [GenBank: NM_000551] following the guidelines from the Human Genetic Variation Society (www.hgvs.org/mutnomen).

\section{In silico analysis}

In silico prediction of the functional consequence of the missense variants was performed using the web-based program Align GVGD [10]. Align GVGD grades of $>=$ C25 were considered likely deleterious. The following three splice site prediction programs were used to predict the variants influence on splicing pattern: www. fruitfly.org/seq_tools/splice.html [11], www.cbs.dtu.dk/ services/NetGene2 [12] and www.umd.be/SSF [13]. The genomic sequence spanning the intron mutations was submitted according to the guidelines of each program and default settings were used in all predictions.

\section{Results and discussion}

Until August 2011, 289 Danish patients had been screened for mutations in the $V H L$ gene by direct sequencing and MLPA analysis. Germ-line mutations were detected in 36 families and the mutations were distributed widely throughout the gene except for the first $\sim 60$ amino acids (Figure 1). The pathogenicity of the 26 different mutations found in the $V H L$ gene were classified using published data and in silico prediction analysis (Table 2).

\section{In-frame deletion, frame-shift and nonsense mutations}

The novel c.342_343dupGGT mutation is an in-frame duplication inserting a glycine residue between glycine-114 
Table 2 VHL mutations identified in Danish patients with VHL suspected disease

\begin{tabular}{|c|c|c|c|c|c|c|}
\hline Family ID & Exon & Nucleotide & Protein & In silico & Interpretation & References \\
\hline 04012 & & c.1-?_340+?del & & & Pathogenic & {$[14,15]$} \\
\hline \multicolumn{7}{|l|}{04013} \\
\hline \multicolumn{7}{|l|}{04231} \\
\hline 00117 & & c.1-?_463+?del & & & Pathogenic & [14] \\
\hline \multicolumn{7}{|l|}{04178} \\
\hline 00108 & 1 & C.194 C> T & p.Ser65Leu & C65 & Pathogenic & {$[14,16]$} \\
\hline 00118 & 1 & c.194 C> G & p.Ser65Trp & C65 & Pathogenic & [14-17] \\
\hline \multicolumn{7}{|l|}{04025} \\
\hline 00116 & 1 & $c .239 \mathrm{G}>\mathrm{T}$ & p.Ser80lle & C15 & Pathogenic & [14-19] \\
\hline 04268 & 1 & c. 257 C > T & p.Pro86Leu & $\mathrm{CO}$ & Pathogenic & [15-17] \\
\hline 00101 & 1 & $c .278 \mathrm{G}>\mathrm{A}$ & p.Gly93Asp & $\mathrm{CO}$ & Pathogenic & {$[14,16]$} \\
\hline 00113 & 1 & $c .293 A>G$ & p.Tyr98Cys & C65 & Pathogenic & {$[15,20]$} \\
\hline 00107 & 1 & c.319 C > T & p.Arg107Cys & $\mathrm{C} 25$ & Pathogenic & Novel \\
\hline 00109 & 1 & c.337 C > T & p.Arg113X & & Pathogenic & {$[21]$} \\
\hline 04283 & & c. $340+5 \mathrm{G}>\mathrm{C}$ & & & Polymorphism & {$[17,22,23]$} \\
\hline 00121 & 1 & c.342_343dupGGT & p.Gly114_His115insGly & & Unknown & Novel \\
\hline 00103 & & c.341-?_463+?del & & & Pathogenic & {$[14,18]$} \\
\hline \multicolumn{7}{|l|}{00105} \\
\hline \multicolumn{7}{|l|}{04208} \\
\hline \multicolumn{7}{|l|}{04078} \\
\hline 00107 & 2 & C.353 T>C & p.Leu118Pro & $\mathrm{C} 25$ & Pathogenic & {$[16,17,24]$} \\
\hline 00102 & 2 & $\mathrm{c} .407 \mathrm{~T}>\mathrm{C}$ & p.Phe136Ser & C55 & Pathogenic & {$[16,17]$} \\
\hline 00104 & 2 & c. 433 C > T & p.Gln145X & & Pathogenic & [17] \\
\hline 04008 & & $c .463+1 \mathrm{G}>\mathrm{T}$ & & & Pathogenic & {$[16,18]$} \\
\hline 04030 & & $c .463+8 C>T$ & & & Unknown & [25] \\
\hline \multicolumn{7}{|l|}{04163} \\
\hline 04135 & & c.464-?_642+?del & & & Pathogenic & {$[14,15]$} \\
\hline \multicolumn{7}{|l|}{04168} \\
\hline \multicolumn{7}{|l|}{04181} \\
\hline \multicolumn{7}{|l|}{04237} \\
\hline 00121 & 3 & c. 481 C > T & p.Arg161X & & Pathogenic & {$[14,16,18,22]$} \\
\hline 04015 & 3 & C. $488 \mathrm{~T}>\mathrm{A}$ & p.Leu163His & $\mathrm{CO}$ & Pathogenic & [22] \\
\hline 00123 & 3 & c. $496 \mathrm{G}>\mathrm{T}$ & p.Val166Phe & $\mathrm{CO}$ & Pathogenic & {$[14,16,17]$} \\
\hline 00106 & 3 & C.499 C > T & p.Arg167Trp & $\mathrm{C} 65$ & Pathogenic & {$[16-18,24]$} \\
\hline 00112 & 3 & c.520_521dupAA & p.Asn174LysfsX29 & & Pathogenic & Novel \\
\hline 04026 & 3 & C. $548 C>A$ & p.Ser183X & & Pathogenic & {$[14,16]$} \\
\hline 04024 & 3 & c.606dupA & p.Gln203ThrfsX53 & & Pathogenic & [17] \\
\hline
\end{tabular}

In silico predictions was performed using the web-based program Align GVGD and grades above 25 was considered likely deleterious.

and histidine-115 (p.Gly114_His115insGly) in the VHL protein. The GGT duplication is located in the 5' end of exon 2 close to the splice acceptor site. However, in silico analysis did not predict any major changes in the splicing pattern and thus the pathogenicity of the p.Gly114_His115insGly mutation remains unknown. Since only the proband was screened it has not been possible to examine if this specific variant co-segregates with VHL disease.
The two frame-shift mutations, c.520_521dupAA (p.Asn174LysfsX28) and c.606dupA (p.Gln203ThrfsX55), both located in exon 3, introduce two new termination codons. The c.520_521dupAA frame-shift leads to a truncated pVHL, whereas the c.606dupA frame-shift results in a larger pVHL. To our knowledge only the c.606dupA mutations has been described before [17], however both mutations are classified as pathogenic. 
The four nonsense mutations found in exon 1 (p.Arg113X), exon 2 (p.Gln145X) and exon 3 (p.Arg161X and p.Ser163X) have previously been reported and are all classified as pathogenic [14,16-18,21,22].

\section{Missense mutations}

Seven of the twelve identified missense mutations were located in exon 1, two in exon 2 and three in exon 3 (Figure 1). Besides these, a known polymorphism (c.74 C > T, p.Pro25Leu) $[19,26]$ was found in six unrelated Danish families.

Two mutations changed the serine-65 residue to leucine (p.Ser65Leu) and tryptophan (p.Ser65Trp), respectively. The p.Ser65Leu mutation was found in several members of the same family; four mutation- or obligate carriers were diagnosed with VHL disease between 27 and 46 years, five carriers were unaffected ( $0-22$ years), two obligate carriers died unaffected ( 85 and 88 years) and the parents of three obligate carriers died unaffected (70 and 92 years). The p.Ser65Trp mutation was found in two unrelated individuals with VHL related disease. Serine-65 mutations are known to disrupt binding of pVHL to HIF-1 $\alpha$ [27] and are observed in several type 1 VHL affected families [14,15,17]. In silico analysis predicted a pathogenic effect (class C65) of both the p.Ser65Leu and the p.Ser65Trp mutations supporting the hypothesis that both mutations are pathogenic. The observation of unaffected mutation carriers may indicate reduced penetrance of the p.Ser65Leu mutation or the co-inheritance of a protective genetic modifier in some family members.

The p.Ser80Ile mutation was found in a single patient. Although in silico analysis classify the mutation of low clinical significance (class C15) the mutation is expected to disrupt the three-dimensional structure of pVHL [19] indicating that the mutation is pathogenic. The mutation has previously been identified in a Hungarian type 2 VHL affected family where all members except the proband's mother showed clinical manifestations of VHL disease [19].

The p.Pro86Leu mutation was found in one individual and the p.Gly93Asp mutation was found in eight members of one family. The mutations have previously been associated with type 1 and type 2 VHL disease, respectively $[14,16,17]$. The two mutations are both located in the $\beta$-domain of pVHL (Figure 1) where the proline-86 residue is important for the structural integrity of the $\beta$ sandwich [6]. Moreover, the p.Pro86Leu mutation was reported as a $V H L$ hotspot $[6,28]$. These findings indicate a pathogenic role of the two missense mutations regardless of the result of the in silico analysis that predicted the mutations to be without clinical significance (class 0$)$.
The p.Tyr98Cys missense mutation was found in two patients from the same family and has previously been associated with type 2 VHL disease [15]. Another germline mutation at tyrosine-98 (p.Tyr98His) was reported as a German founder mutation [20]. The tyrosine-98 residue is located on the surface of the $\beta$-sandwich [6] and in silico analysis predicted the mutation to be of high clinical significance (class C65).

Both a mother and her son were found to carry two VHL missense mutations (p.Arg107Cys and p.Leu118Pro) proving that both mutations are present on the same allele. Changes of the arginine-107 residue to glycine and histidine have previously been reported $[18,29]$ whereas the p.Arg107Cys missense mutation is novel. The p.Leu118Pro mutation has been associated with type 1 VHL disease [24]. Hence, clinical findings and Align GVGD classifications (class C25) indicates a pathogenic role of both mutations although the contribution of each mutation to the phenotype is unknown.

The p.Phe136Ser mutation was found in a single patient. This mutation has previous been associated with type 1 VHL disease [16,17] and together with in silico analysis (class C55) this suggest that the mutation is pathogenic.

The p.Leu163His, p.Val166Phe and p.Arg167Trp mutations are all located in the $\alpha$-domain of $\mathrm{pVHL}$ (Figure 1). In silico analysis does not indicate a pathogenic effect of p.Leu163His and p.Val166Phe (class C0). However, Leucine-163 and valine-166 are contact residues when pVHL binds to elongin C [30] and mutations at the leucine-163 residue have shown to impair the ability of pVHL to target HIF-1 $\alpha$ for destruction [31]. These findings suggest that both mutations are pathogenic. Arginine-167 has a structural role in stabilizing the $\mathrm{H} 1$ helix and the $\alpha-\beta$ domain interface and was previously reported as the most frequently mutated residue in VHL [6]. The importance of arginine-167 is supported by in silico analysis classifying the p.Arg167Trp mutation as pathogenic (class C65).

\section{Intronic mutations}

We identified three intronic mutations located in intron 1 $($ c.340 $+5 \mathrm{G}>\mathrm{C})$ and intron $2(\mathrm{c} .463+1 \mathrm{G}>\mathrm{T}$ and c. $463+8 \mathrm{C}>\mathrm{T})$. The c. $340+5 \mathrm{G}>\mathrm{C}$ intronic mutation was previously observed in a VHL family in trans with a pathogenic $V H L$ mutation [22,23] suggesting it is a benign polymorphism. This is supported by in silico analysis showing no major changes in splicing pattern. The c. $463+1 \mathrm{G}>\mathrm{T}$ intronic mutation disrupt the highly conserved splice donor site [32] and in silico analysis predicted skipping of $V H L$ exon 2. Combined with an earlier described association with type 1 VHL disease [18] this clearly suggests a pathogenic effect of this mutation. The c. $463+8 \mathrm{C}>\mathrm{T}$ mutation was found in two unrelated 
individuals. The mutation was previously described as a variant of unknown significance [25]. In silico analysis suggested no major changes in the splicing pattern indicating that the variant might be a benign polymorphism. However, functional studies or analysis of co-segregation are needed to confirm this.

\section{Large genomic rearrangements}

Large genomic rearrangements were identified in thirteen Danish families. Deletion of exon 1 (c.1-?_340+ ?del) was found in three families, deletion of both exon 1 and 2 (c.1-?.463+?del) was found in two families whereas deletion of either exon 2 (c.341-?.463+?del) or exon 3 (c.464-?_642+?del) was found in four families each. Although the exact break point of the four different deletions is unknown similar deletions have previously been reported as pathogenic [14,15,18]. Large genomic rearrangements leading to truncated versions of pVHL are primarily associated with type 1 VHL disease [33].

Altogether, we found $33.4 \%$ missense mutations, $11.1 \%$ nonsense mutations, $11.1 \%$ intronic mutations, $2.7 \%$ inframe mutations, 5.6\% frame-shift mutations and 36.1\% large genomic rearrangement. This spectrum of mutations is in concordance with a recently published study [34], although we found less missense mutations (33.4\% vs $52 \%$ ) and a higher prevalence of large genomic rearrangements ( $36.1 \%$ vs $11 \%)$.

\section{Conclusions}

In conclusion, we have screened 289 Danish probands with VHL suspected disease and identified 26 mutations in 36 families. We classified all large genomic rearrangements and all nonsense- and missense mutations as pathogenic. The two frame-shift mutations were classified as pathogenic, whereas the in-frame duplication was classified as a variant of unknown significance. Of the three intron variants found, one was a benign polymorphism, one was pathogenic and one was a variant of unknown significance. Of these mutations the missense mutation c.319 C > T (p.Arg107Cys), the in-frame duplication c.342_343dupGGT (p.GlyHis_His115insGly) and the frame-shift mutation c.520_521dupAA (p.Asn174LysfsX29) were found to be novel.

\section{Competing interests}

The authors declare that they have no competing interests.

\section{Authors' contributions}

$\mathrm{TVOH}$ and LFH designed the study and LS was involved in recruitment of patients. MD and TVOH performed the in silico analyses. MD, TVOH, FCN, LS and LFH have been involved in drafting the manuscript or revising it critically for important intellectual content. All authors read and approved the final version of the manuscript.

\section{Acknowledgements}

We thank Vibeke Tranbohus and Aseeba Ajub for expert technical help in screening of the VHL gene. Moreover, we thank the Danish VHL group for collaboration and all patients and families for their participation.

\section{Author details}

${ }^{1}$ Center for Genomic Medicine, Rigshospitalet, Copenhagen University Hospital, Copenhagen, Denmark. ²Department of Clinical Genetics, Aarhus University Hospital, Aarhus, Denmark. ${ }^{3}$ Department of Clinical Genetics, Aalborg Hospital, Aalborg, Denmark.

Received: 25 January 2012 Accepted: 29 June 2012

Published: 16 July 2012

\section{References}

1. Maher ER: Von Hippel-Lindau disease. Curr Mol Med 2004, 4:833-842.

2. Kaelin WG Jr: Molecular basis of the VHL hereditary cancer syndrome. Nat Rev Cancer 2002, 2:673-682.

3. Schoenfeld A, Davidowitz EJ, Burk RD: A second major native von HippelLindau gene product, initiated from an internal translation start site, functions as a tumor suppressor. Proc Natl Acad Sci U S A 1998, 95:8817-8822.

4. Iliopoulos O, Ohh M, Kaelin WG Jr: pVHL19 is a biologically active product of the von Hippel-Lindau gene arising from internal translation initiation. Proc Natl Acad Sci U S A 1998, 95:11661-11666.

5. Blankenship C, Naglich JG, Whaley JM, Seizinger B, Kley N: Alternate choice of initiation codon produces a biologically active product of the von Hippel Lindau gene with tumor suppressor activity. Oncogene 1999, 18:1529-1535

6. Stebbins CE, Kaelin WG Jr, Pavletich NP: Structure of the VHL-ElonginCElonginB complex: implications for VHL tumor suppressor function. Science 1999, 284:455-461.

7. Poulsen ML, Budtz-Jorgensen $E$, Bisgaard ML: Surveillance in von HippelLindau disease (vHL). Clin Genet 2010, 77:49-59.

8. Poulsen ML, Gimsing S, Kosteljanetz M, Moller HU, Brandt CA, Thomsen C, Bisgaard ML: von Hippel-Lindau disease: Surveillance strategy for endolymphatic sac tumors. Genet Med 2011, 13:1032-1041.

9. von Hippel-Lindau reference program. http://www.ugeskriftet.dk/portal/page/ portal/LAEGERDK/UGESKRIFT_FOR_LAEGER/KLINISKE_VAERKTOEJER/ KLARINGSRAPPORTER/Von_Hippel-Lindaus_Sygdom.pdf.

10. Tavtigian SV, Deffenbaugh AM, Yin L, Judkins T, Scholl T, Samollow PB, de SD, Zharkikh A, Thomas A: Comprehensive statistical study of 452 BRCA1 missense substitutions with classification of eight recurrent substitutions as neutral. J Med Genet 2006, 43:295-305.

11. Reese MG, Eeckman FH, Kulp D, Haussler D: Improved splice site detection in Genie. J Comput Biol 1997, 4:311-323.

12. Hebsgaard SM, Korning PG, Tolstrup N, Engelbrecht J, Rouze P, Brunak S: Splice site prediction in Arabidopsis thaliana pre-mRNA by combining local and global sequence information. Nucleic Acids Res 1996, 24:3439-3452.

13. Yeo G, Burge CB: Maximum entropy modeling of short sequence motifs with applications to RNA splicing signals. J Comput Biol 2004, 11:377-394

14. Hes FJ, van der Luijt RB, Janssen AL, Zewald RA, de Jong GJ, Lenders JW Links TP, Luyten GP, Sijmons RH, Eussen HJ, Halley DJ, Lips CJ, Pearson PL, van den Ouweland AM, Majoor-Krakauer DF: Frequency of Von HippelLindau germline mutations in classic and non-classic Von Hippel-Lindau disease identified by DNA sequencing, Southern blot analysis and multiplex ligation-dependent probe amplification. Clin Genet 2007, 72:122-129.

15. Zhang J, Huang Y, Pan J, Liu D, Zhou L, Xue W, Chen Q, Dong B, Xuan H: Germline mutations in the von Hippel-Lindau disease $(\mathrm{VHL})$ gene in mainland Chinese families. J Cancer Res Clin Oncol 2008, 134:1211-1218.

16. Zbar B, Kishida T, Chen F, Schmidt L, Maher ER, Richards FM, Crossey PA, Webster AR, Affara NA, Ferguson-Smith MA, Brauch H, Glavac D, Neumann HP, Tisherman S, Mulvihill JJ, Gross DJ, Shuin T, Whaley J, Seizinger B, Kley N, Olschwang S, Boisson C, Richard S, Lips CH, Lerman M: Germline mutations in the Von Hippel-Lindau disease (VHL) gene in families from North America, Europe, and Japan. Hum Mutat 1996, 8:348-357.

17. Ong KR, Woodward ER, Killick P, Lim C, Macdonald F, Maher ER: Genotypephenotype correlations in von Hippel-Lindau disease. Hum Mutat 2007, 28:143-149. 
18. Siu WK, Ma RC, Lam CW, Mak CM, Yuen YP, Lo FM, Chan KW, Lam SF, Ling SC, Tong SF, So WY, Chow CC, Tang MH, Tam WH, Chan AY: Molecular basis of von Hippel-Lindau syndrome in Chinese patients. Chin Med J (Engl) 2011, 124:237-241.

19. Patocs A, Gergics P, Balogh K, Toth M, Fazakas F, Liko I, Racz K: Ser80lle mutation and a concurrent Pro25Leu variant of the VHL gene in an extended Hungarian von Hippel-Lindau family. BMC Med Genet 2008, 9:29.

20. Brauch H, Kishida T, Glavac D, Chen F, Pausch F, Hofler H, Latif F, Lerman MI, Zbar B, Neumann HP: Von Hippel-Lindau (VHL) disease with pheochromocytoma in the Black Forest region of Germany: evidence for a founder effect. Hum Genet 1995, 95:551-556.

21. Peyre M, Gaillard S, van Effenterre R, Giraud S, Richard S: Conservative management of endolymphatic sac tumors in von Hippel-Lindau disease: case report. Acta Neurochir 2011, 153:42-47.

22. Young AC, Craven RA, Cohen D, Taylor C, Booth C, Harnden P, Cairns DA, Astuti D, Gregory W, Maher ER, Knowles MA, Joyce A, Selby PJ, Banks RE: Analysis of VHL Gene Alterations and their Relationship to Clinical Parameters in Sporadic Conventional Renal Cell Carcinoma. Clin Cancer Res 2009, 15:7582-7592.

23. Erlic Z, Hoffmann MM, Sullivan M, Franke G, Peczkowska M, Harsch I, Schott M, Gabbert HE, Valimaki M, Preuss SF, Hasse-Lazar K, Waligorski D, Robledo M, Januszewicz A, Eng C, Neumann HP: Pathogenicity of DNA variants and double mutations in multiple endocrine neoplasia type 2 and von Hippel-Lindau syndrome. J Clin Endocrinol Metab 2010, 95:308-313.

24. Zhou MI, Wang H, Foy RL, Ross JJ, Cohen HT: Tumor suppressor von Hippel-Lindau (VHL) stabilization of Jade-1 protein occurs through plant homeodomains and is VHL mutation dependent. Cancer Res 2004, 64:1278-1286.

25. Brauch $H$, Hoeppner W, Jahnig H, Wohl T, Engelhardt D, Spelsberg F, Ritter $\mathrm{MM}$ : Sporadic pheochromocytomas are rarely associated with germline mutations in the vhl tumor suppressor gene or the ret protooncogene. J Clin Endocrinol Metab 1997, 82:4101-4104.

26. Pettman RK, Crowley A, Riddell C, Ludman MD: VHL P25L is not a pathogenic von Hippel-Lindau mutation: a family study. Mol Diagn Ther 2006, 10:239-242.

27. Miller F, Kentsis A, Osman R, Pan ZQ: Inactivation of VHL by Tumorigenic Mutations That Disrupt Dynamic Coupling of the PVHL-Hypoxiainducible Transcription Factor-1alfa Complex. J Biol Chem 2005, 280:7985-7996.

28. Richards FM: Molecular pathology of von Hippel-Lindau disease and the VHL tumour suppressor gene. Expert Rev Mol Med 2001, 3:1-27.

29. Boedeker CC, Erlic Z, Richard S, Kontny U, Gimenez-Roqueplo AP, Cascon A, Robledo M, de Campos JM, van Nederveen FH, de Krijger RR, Burnichon N, Gaal J, Walter MA, Reschke K, Wiech T, Weber J, Rückauer K, Plouin PF, Darrouzet V, Giraud S, Eng C, Neumann HPH: Head and Neck Paragangliomas in Von Hippel-Lindau Disease and Multiple Endocrine Neoplasia Type 2. J Clin Endocrinol Metab 2009, 94:1938-1944.

30. Botuyan MV, Mer G, Yi GS, Koth CM, Case DA, Edwards AM, Chazin WJ, Arrowsmith $\mathrm{CH}$ : Solution structure and dynamics of yeast elongin $\mathrm{C}$ in complex with a von Hippel-Lindau peptide. J Mol Biol 2001, 312:177-186.

31. Wiesener MS, Seyfarth M, Warnecke C, Jurgensen JS, Rosenberger C, Morgan NV, Maher ER, Frei U, Eckardt KU: Paraneoplastic erythrocytosis associated with an inactivating point mutation of the von Hippel-Lindau gene in a renal cell carcinoma. Blood 2002, 99:3562-3565.

32. Chong A, Zhang G, Bajic VB: Information for the Coordinates of Exons (ICE): a human splice sites database. Genomics 2004, 84:762-766.

33. Barry RE, Krek W: The von Hippel-Lindau tumour suppressor: a multifaceted inhibitor of tumourigenesis. Trends Mol Med 2004, 10:466-472.

34. Nordstrom-O'Brien M, van der Luijt RB, van Rooijen E, van den Ouweland AM, Majoor-Krakauer DF, Lolkema MP, van Brussel A, Voest EE, Giles RH: Genetic analysis of von Hippel-Lindau disease. Hum Mutat 2010, 31:521-537.

doi:10.1186/1471-2350-13-54

Cite this article as: Dandanell et al:: Identification of 3 novel VHL germline mutations in Danish VHL patients. BMC Medical Genetics 2012 13:54. 